\title{
USAGE OF CONTROL CHARTS FOR TIME SERIES ANALYSIS IN FINANCIAL MANAGEMENT
}

\author{
Martin KOVÁŘÍK ${ }^{1}$, Libor SARGA ${ }^{2}$, Petr KLÍMEK ${ }^{3}$ \\ Department of Statistics and Quantitative Methods, Faculty of Management and Economics, \\ Tomas Bata University, Mostni 5139, 76001 Zlín, Czech Republic \\ E-mails: ${ }^{1}$ kovarik.fame@seznam.cz; ${ }^{2}$ sarga@fame.utb.cz (corresponding author); \\ 3klimek@fame.utb.cz
}

Received 10 July 2012; accepted 17 September 2012

\begin{abstract}
We will deal with corporate financial proceeding using statistical process control, specifically time series control charts. The article outlines intersection of two disciplines, namely econometrics and statistical process control. Theoretical part discusses methodology of time series control charts, and in research part, the methodology is demonstrated on two case studies. The first focuses on analysis of Slovak currency from the perspective of its usefulness for generating profits through time series control charts. The second involves regulation of financial flows for a heteroskedastic financial process by EWMA and ARIMA control charts. We use Box-Jenkins methodology to find models of time series of annual Argentinian Gross Domestic Product available as a basic index from 1951-1998. We demonstrate the versatility of control charts not only in manufacturing but also in managing financial stability of cash flows. Specifically, we show their sensitivity in detecting even small shifts in mean which may indicate financial instability. This analytical approach is widely applicable and therefore of theoretical and practical interest.
\end{abstract}

Keywords: statistical process control, Shewhart's control charts, autocorrelation, control chart EWMA, control chart CUSUM, control chart ARIMA.

JEL CLassification: C39, C49, C59.

\section{Introduction}

Traditional statistical process control (SPC) schemes, such as Shewhart and cumulative sum (CUSUM), control charts assume data collected from processes independent. However, the assumption has been challenged as it has been found they are serially correlated in many practical situations. Performance of traditional control charts deteriorates significantly under autocorrelation which motivated the pioneering work by Alwan (1992), who proposed the monitoring of forecasted errors after appropriate time series model has been fitted toa process. The method is intuitive as autocorrelation can be accounted for by the underlying time series model while the residual component 
captures process' independent random errors. Traditional SPC schemes can be applied to monitor residuals.

Subsequent work on this problem can be broadly classified into two themes: time series models based and model-free. For the former, three general approaches have been proposed: those which monitor residuals, those based on direct observations, and those based on new statistics. Their brief account is presented in this chapter.

The time series model based approach is easy to understand and effective in some situations. However, it requires identifying appropriate time series model from a set of initial in-control data. This may not be easy to establish it in practice and may be too complicated for practicing engineers. Hence, the model-free approach has recently attracted much attention (Kovarik, Klimek 2013; Kovarik, Sarga 2014).

The most popular model-free approach is to form a multivariate statistic from the autocorrelated univariate process, and subsequently monitor it with corresponding multivariate control chart (Gervini 2003). Krieger et al. (1992) used a multivariate CUSUM scheme. Apley and Tsung (2002) adapted the $T^{2}$ control chart for monitoring univariate autocorrelated processes. Atienza et al. (1997) proposed Multivariate boxplot- $T^{2}$ control chart. Dyer et al. (2003) adapted the use of multivariate EWMA control chart for autocorrelated processes. Another model-free approach is to use batch means control charts as proposed by Runger and Willemain (1996), referenced by Montgomery and Mastrangelo (1991), and discussed in detail by Sun and Xu (2004). The main advantage of this approach lies in its simplicity. In an attempt to "break" dependency, it simply divides sequential observations into a number of batches and monitors the batches' means on a standard individuals control chart.

Statistical financial flow proceeding denotes corporate cash flow management. We can avoid losses caused by undelivered goods, bad financial investment etc. by cash flow monitoring. Such financial analysis should be done once a year.

Case study 1 analyses Slovak currency in the 2000-2008 period from the perspective of its usefulness for generating profits for company management through time series control charts (Kovarik 2012, 2013a, 2013b). On the basis of time series O/N analysis of Slovak crown currency rates and its description via Box-Jenkins methodology for random processes modeling and subsequent implementation of this mathematical vehicle into regulation diagrams for time series, the authors uncover the potential of Slovak crown to provide enough space for generating substantial profits. In the case study, EWMA and CUSUM control chart for volatility change point detection will be used. The end of the paper will be dedicated to the ARIMA and EWMA control charts together with practical examples of autocorrelated data for mean shift detection (see Case Study 2). Box-Jenkins methodology for finding models of time series of Argentina's annual gross domestic product will be used which is available as a basic index from 1951 to $1998(1995=100)$. Based on the selected model, we will calculate the predictions of the time series of 4 observations and on the basis of a suitable ARIMA model construct control charts for volatility change point detection. 


\section{Fundamental data assumptions}

This part primarily focuses on crucial problems with statistical analysis data assumptions. Fundamental assumptions for statistical process regulation can be described as:

- data normality, symmetry,

- constant mean of the process,

- constant variance (standard deviation) of data,

- independence, no autocorrelation in data,

- absence of outliers (Meloun, Militký 2006).

Most data analysis processes and their conclusions are dependent on fulfilling several conditions. If violated, all other calculations of means, confidence intervals, quantiles, statistical tests, Shewhart's charts, and capability indices are questionable and not really correct as they usually offer incorrect and inaccurate results and conclusions. Therefore we should be very careful about the abovementioned conditions (data normality, symmetry, etc.). Their violations in application of regulation by Shewhart's charts in different technologies are displayed in Table 1.

Table 1. Typical violations of assumptions for the application of regulation by Shewhart's charts in different technologies

\begin{tabular}{lcccc}
\hline Branch, technology (value) & Normality & Independence & $\begin{array}{c}\text { Constant } \\
\text { mean }\end{array}$ & Homogenity \\
\hline Machinery (size) & YES & YES & YES & YES \\
\hline Mechanical testing (strength, flexibility) & NO & YES & YES & NO \\
\hline $\begin{array}{l}\text { Chemistry, metalurgy, metals } \\
\text { (concentration levels) }\end{array}$ & YES & NO & NO & NO \\
\hline Environment (concentration), energetics & NO & NO & NO & NO \\
\hline Electrical quantities, components & YES & YES & YES & NO \\
\hline Biochemistry, pharmacy, food & NO & NO & YES & YES \\
\hline Internal economic and financial indicators & NO & NO & NO & YES \\
\hline Sociology, human resources & NO & NO & NO & NO \\
\hline
\end{tabular}

Source: Meloun, Militký (2006).

The conditions should be verified by means of statistical tests. For example, data asymmetry should be expected in physical quantities such as strength or viscosity, strong autocorrelation (dependence) in continuous processes in chemistry, pharmacy, food and metals. Quality of input process material can result in mean shifting while not normally distributed data can be often seen in ecologic processes. There, data are very asymmetric, usually following lognormal distribution. As mentioned previously, a list of typical data assumptions corruptions for Shewhart's chart in different applications are discussed in Table 1 (Meloun, Militký 2006). 


\section{Literature review}

\subsection{Control charts CUSUM}

CUSUM control charts were introduced by Page in 1954 based on cumulative sums. Their main advantage is very quick detection of relatively small shifts in the process' mean significantly faster than by the Shewhart's control charts.

The sequential sums of deviations from $\mu_{0}$ are used for the CUSUM control charts' construction. If $\mu_{0}$ is a target value for population mean and $X_{j}$ a sample mean then the CUSUM control chart is constructed by plotting variables of the $S_{i}=\sum_{j=1}^{i}\left(X_{j}-\mu_{0}\right)$
type. The process is called a random walk (Harris, Ross 1991).

\subsection{CUSUM - chart for individual values and for samples means from normally distributed data}

Values of $x_{i}$ are independent with the same normal distribution $N\left(\mu, \sigma^{2}\right)$ with known population mean and known population standard deviation $\sigma$. We assume logical subgroups with the same volume $n$. Cumulative sum - CUSUM $C_{n}$ is defined for individual values $(n=1)$ as:

A) on a base of original scale:

$$
C_{n}=\sum_{j=1}^{n}\left(x_{j}-\mu\right)
$$

B) on a base of normal distribution where mean $=0$ and standard deviation $=1$ :

$$
U_{j}=\frac{\left(x_{j}-\mu\right)}{\sigma}, S_{n}=\sum_{j=1}^{n} U_{i} .
$$

The CUSUM $C_{n}$ is almost the same as CUSUM $S_{n}$ measured in the units of standard deviation $\sigma$. Equation for $C_{n}$ can be written recurrently (Chandra 2001): $C_{0}=0, C_{n}=$ $C_{n-1}+\left(x_{n}-\mu\right)$; and using the same principle for $S_{n}: S_{0}=0, S_{n}=S_{n-1}+U_{n}$.

Suppose the original distribution of observed variable $N\left(\mu, \sigma^{2}\right)$ changes into $N(\mu+\delta$, $\sigma^{2}$ ) distribution for integer $t$ (at an arbitrary moment). Therefore, the population mean $\mu$ will face an arbitrary shift of $\delta$.

It also means the shift starts at point $\left(m, C_{m}\right)$, growing linearly with the slope $\delta$. However, population means shift can be more complicated. The CUSUM control chart can reflect all these changes (Harris, Ross 1991).

\subsection{CUSUM for sample means $\overline{\boldsymbol{x}}_{\boldsymbol{j}}$}

Until now, we have considered mainly individual values. Now, suppose we have subgroups with $m$ observations and calculating sample means from this subgroups. We have to work with the sample mean standard deviation $\sigma_{\bar{x}}=\frac{\sigma}{\sqrt{m}}$. In this case, a shift of mean $\Delta$ will not be measured in units of $\sigma$ but instead in units of $\sigma_{\bar{x}}$. In the abovementioned formulas, individual values of $x_{i}$ will be substituted with sample means $\bar{x}_{j}$ and process standard deviation $\sigma$ with sample mean standard deviation $\sigma_{\bar{x}}$ (Lu, Reynolds 1999a). 


\section{New process mean estimate}

If there is a shift, a new process mean may be estimated from the formula:

$$
\hat{\mu}=\left\{\begin{array}{l}
\mu_{0}+\mathrm{K}+\frac{\mathrm{C}_{\mathrm{I}}^{+}}{\mathrm{N}^{+}} \text {for } \mathrm{C}_{\mathrm{I}}^{+}>\mathrm{H} \\
\mu_{0}-\mathrm{K}+\frac{\mathrm{C}_{\mathrm{I}}^{-}}{\mathrm{N}^{-}} \text {for } \mathrm{C}_{\mathrm{I}}^{-}<-\mathrm{H}
\end{array},\right.
$$

where $\mathrm{N}^{+}$and $\mathrm{N}^{-}$are numbers of selected points in a moment (Chambers, Wheeler 1992), when $C_{n}^{+}=0$, or when $C_{n}^{-}=0$, respectively.

\section{Comparison of CUSUM and Shewhart's control charts}

The example practically shows sensitivity of the CUSUM control chart in comparison with Shewhart's control chart for sample means. CUSUM control chart detects process mean deviation towards the lower values (around subgroup 20 - see Figure 1) while Shewhart's control chart does not detect this deviation (Harris, Ross 1991). It does not even detect a shift towards the upper values (around subgroup 56). It only detects a big shift around subgroup 70 (see Figs 1 and 2) (Lu, Reynolds 1999b).

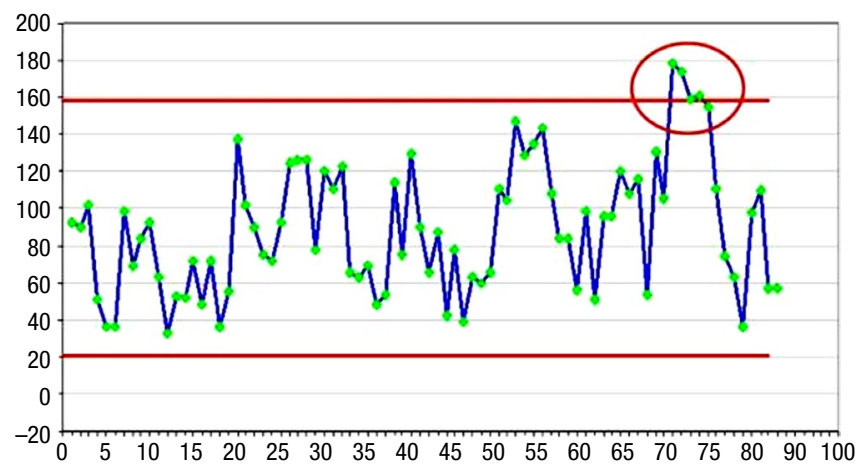

Fig. 1. Shewhart's control chart Source: QC Expert $2.5 \mathrm{Cz}$.

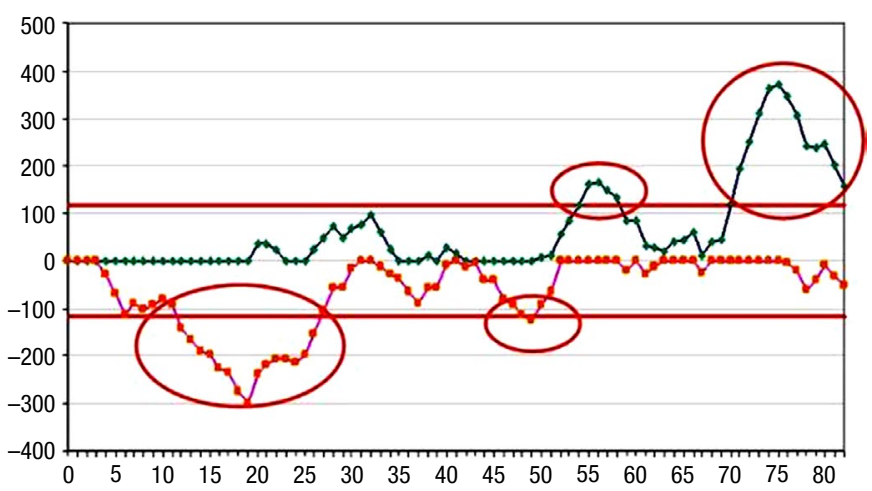

Fig. 2. CUSUM control chart Source: QC Expert $2.5 \mathrm{Cz}$. 


\subsection{Dynamic control chart EWMA}

EWMA (Exponentially Weighted Moving Average) dynamic control charts are used when the following conditions are met:

- no dependency with positive autocorrelation,

- mean is not constant, slow changes (Montgomery, Friedman 1989).

A sudden change in mean will only cause a control limits crossing. These dynamic charts not only provide information about the "in control" process but also about the process' dynamic development. As mentioned previously, we consider solely data which are independent with positive autocorrelation. If the measured observations are influenced by previous ones we can say that they are dependent. A special case of such dependence is called autocorrelation of first degree when this dependence is linear. If there is a positive autocorrelation in data, then smaller value follows a smaller value and higher value follows a higher value. Data thus have tendency to preserve their original values. Process is not stable in case of negative autocorrelation. If there is a negative autocorrelation in data, higher value follows a smaller value and smaller value follows a higher value.

Suppose that we measure values $x_{1}, x_{2}, x_{3}, \ldots$ for the variable $\mathrm{X}$ in the process. We use so called one-step predictions $\hat{x}_{k}$ to construct the $C L, U C L$, and $U C L$ for the control chart. The predictions are determined from the following equation $\hat{x}_{k}=\hat{x}_{k-1}+\lambda e_{k}$ for $k=1,2,3, \ldots$, where the starting prediction value $\hat{x}_{0}$ is equal to the target value of $\mu_{0}$. Parameter $\lambda$ (level of "forgetting") is calculated by trying where the function $\sum_{k=1}^{n} e_{k}^{2}$ is minimal. Number $n$ is equal to number of measured values of regulated variable. It is recommended that $n$ is greater than 50. If the error values of one-step prediction of $e_{k}$ for the optimal value of parameter $\lambda$ are not correlated and if they have a normal distribution then the center line $C L_{k}$, control limits $U C L_{k}$ and $L C L_{k}$ for the dynamic control chart EWMA are calculated from the following formulas (Lu, Reynolds 1999a):

$$
C L_{k}=\hat{x}_{k-1}, \quad L C L_{k}=\hat{x}_{k-1}-\hat{\sigma}_{p} u_{1-\frac{\alpha}{2}}, U C L_{k}=\hat{x}_{k-1}+\hat{\sigma}_{p} u_{1-\frac{\alpha}{2}}, \quad \hat{\sigma}_{p}^{2}=\frac{1}{n-1} \sum_{k-1}^{n} e_{k}^{2},
$$

where $\hat{\sigma}_{p}^{2}$ is a standard deviation of $e_{k}$ estimate, and $e_{k}$ values are determined for the optimal value of parameter $\lambda$ (Yourstone, Montgomery 1991).

\subsection{ARIMA control chart}

Classical Shewhart SPC concept assumes the measured data are not autocorrelated. Even very low degree of autocorrelation causes failure of Classical Shewhart control charts exhibited by large number of points outside the regulatory limits in control diagram. This phenomenon is not unique in case of continuous processes where the autocorrelation data given by the inertia processes in time (chemical processes, climate processes etc.). Autocorrelation of data becomes increasingly frequent phenomenon in discrete processes, particularly manufacturing with short production cycles, high-speed production with high degree of production automation and also in test and control operations. In these conditions, it is possible to obtain data about each product with the consequence 
that the interval between measurements (recording) of two consecutive values of the monitored variables is very short. One of the ways to tackle autocorrelated data is the concept of stochastic modelling of time series using Autoregressive Integrated Moving Average (ARIMA) models. The concept of linear stochastic autoregressive models (models AR), moving average (model MA), mixed models (the ARMA models), and ARIMA models based on Box-Jenkins methodology is seen as a time series realization of stochastic process. Box-Jenkins methodology represents a modern concept analysis of stationary and nonstationary time series based on probability theory.

Linear models AR, ARMA and MA are modelling tools for stationary processes. They have characteristic shape of the Autocorrelation Function (ACF) and Partial Autocorrelation Function (PACF), both of which are essential for providing information about the stochastic process. ACF and PACF estimates are used to identify the time series model. Very often, there are nonstationary processes to be found in practice. Nonstationarity can be present due to mean value changing over time or process variance changing over time. If, after the transformation of nonstationary process variance of "random walk" (so-called integrated process) using differential of $d$-th order is the final process model to describe the stationary $\operatorname{ARMA}(p, q)$, the original integrated process is called an autoregressive integrated moving average process of order $p, d$, and $q$, i.e., ARIMA $(p, d, q)$ (Noskievičová 2008).

ARIMA control chart is based on the principle of finding a suitable time series model and use of control chart for residuals (deviations from the values actually measured from calculated values using the model).

A general shape of the model $\operatorname{ARIMA}(p, d, q)$ is:

where:

$$
\Phi_{p}(B) \cdot \nabla^{d} \cdot x_{t}=\Theta_{q}(B) \varepsilon_{t},
$$

$\Phi_{p}(B)=\left(1-\phi_{1} B-\phi_{2} B^{2} \ldots-\phi_{p} B^{p}\right)$ is autoregressive polynomial of $p$-th order, $\Theta_{q}(B)=\left(1-\theta_{1} B-\theta_{2} B^{2} \ldots-\theta_{q} B^{q}\right)$ is moving averages polynomial of $q$-th order, where $\nabla$ represents backward difference (introduced when the model exhibits nonstationarity of the process), $d$ difference order, $t$ time, $B$ back shift operator $B \cdot x_{t}=\left(x_{t-1}\right)$,

$\phi_{1}, \phi_{2}, \ldots, \phi_{p}$ - parameters of autoregressive model, $\theta_{1}, \theta_{2}, \ldots, \theta_{q}-$ parameters of moving averages model,

$\varepsilon_{t}$ is called white noise (unpredictable. normally-distributed fluctuations in the data with zero mean and constant variance, its values are uncorrelated).

If $\hat{x}_{t}$ is an estimate of empirical value of $x_{t}$ calculated using the selected ARIMA model, residuals of this model $e_{t}=x_{t}-\hat{x}_{t}$ will be uncorrelated, normally-distributed random variables.

ARIMA models are the most commonly used in practical applications. Let us consider the model:

$$
x_{t}=\xi+\phi x_{t-1}+\varepsilon_{t},
$$

where $\xi$ a $\varphi(-1<\phi<1)$ are unknown constants and $\varepsilon_{t}$ is normally distributed and uncorrelated variable with zero mean and constant standard deviation $\sigma$. It is called 
autoregressive model of the first order and denoted as AR (1). The values of the reference mark of quality, mutually shifted of $k$ time periods $\left(x_{t}\right.$ and $\left.x_{t-k}\right)$ have the correlation coefficient $\varphi^{k}$. The autocorrelation function should thus fall exponentially. If we expand the previous equation to the form:

$$
x_{t}=\xi+\phi_{1} x_{t-1}+\phi_{2} x_{t-2}+\varepsilon_{t},
$$

we obtain a second-order autoregressive model AR (2). Generally, variable $x_{t}$ is directly dependent on the preceding values $x_{t-1}, x_{t-2}$, etc. in the autoregressive model AR $(p)$. If we model data dependence using the random component $\varepsilon_{t}$, we get moving average model MA $(q)$. First-order moving average model takes a form:

$$
x_{t}=\mu+\varepsilon_{t}-\theta \varepsilon_{t-1} .
$$

There is some correlation between $x_{t}$ and $x_{t-1}$, described as follows: $\rho_{1}=-\theta /\left(1+\theta^{2}\right)$. This corresponds to the shape of the ACF (Noskievičová 2008). When modelling practical problems, it is often suitable to model a compound containing both autoregressive and moving averages component, generally known as ARMA $(p, q)$ (Hušek 2007). Firstorder ARMA model, i.e., ARMA $(1,1)$ is described by the equation:

$$
x_{t}=\xi+\phi x_{t-1}+\varepsilon_{t}-\theta \varepsilon_{t-1} .
$$

It is suitable for chemical and other continuous processes where many quality characteristics can be easily modelled by AR (1). Measurement errors are described by random component which we assume to be random and uncorrelated. The ARMA model also assumes stationary process, i.e., the character quality reference values are around stable mean. Unfortunately, in practice there are processes (e.g., chemical industry), where the values of monitored variable are "running away". In such cases it is convenient to model processes using appropriate model with the backward difference operator $\nabla$, such as ARIMA model $(0,1,1)$ with the formula:

$$
x_{t}=x_{t-1}+\varepsilon_{t}-\theta \varepsilon_{t-1} .
$$

ARIMA models are different from Shewhart model $\left(x_{t}=\mu+\varepsilon_{t}\right.$ for $\left.t=1,2, \ldots\right)$. However, if we put $\varphi=0$ in the equation $x_{t}=\xi+\phi x_{t-1}+\varepsilon_{t}$ or $\theta=0$ in the equation $x_{t}=\mu+\varepsilon_{t}-\theta \varepsilon_{t-1}$, we obtain Shewhart model process. Another important step in the use of ARIMA models is the choice of the appropriate SPC control chart. When residuals testing determines they are not autocorrelated and come from a normal distribution, it is possible to verify whether or not the process is statistically stable. Because the number of observations is equal to one (original empirical values $x_{t}$ were detected by each unit), control charts have priority for individual values and moving range. Location of the mean value $C L$ and upper and lower control limits (UCL, LCL) for the ARIMA chart for individual values can be determined from the formula:

$$
\begin{gathered}
C L=\bar{e}(\cong 0), \\
U C L=\bar{e}+\frac{3}{1,128} \bar{R}_{k l}, \\
L C L=\bar{e}-\frac{3}{1,128} \bar{R}_{k l},
\end{gathered}
$$


where: $\bar{e}$ is average value of residuals; $\bar{R}_{k l}$ is average moving range.

Values $C L, U C L$ and $L C L$ are calculated as follows:

$$
\begin{gathered}
C L=\bar{R}_{k l}, \\
U C L=3,267 \cdot \bar{R}_{k l}, \\
L C L=0 .
\end{gathered}
$$

To increase sensitivity of ARIMA control charts it is recommended to use two-sided CUSUM control chart with the decision interval $\pm H$ or standard EWMA chart, both applied to the model's residuals. If we pursue more quality characteristics simultaneously on a single or multiple products, we can apply Hotelling chart, CUSUM or EWMA charts for more variables to ARIMA models residuals (Noskievičová 2008).

\section{Results of the case studies}

\subsection{Research method conducted}

As the paper's title explains, primary method used for achieving its goals is case studies research. The purpose of the studies is to illustrate time series charts' sensitivity in detecting small shifts. We will exploit the fact that these control charts can be used in certain situations where the data are autocorrelated. The methodology combines quantitative data analysis with financial process simulation which benefits the reliability of conclusions.

\subsection{Case study No 1: Use of CUSUM and EWMA control charts}

Let us analyze a time series of $\mathrm{O} / \mathrm{N}$ rate of the Slovak crown for the 2000-2008 period (Kovarik, Kral 2011). For this purpose the ARIMA model will be used, applied when the resulting process is exhibiting autocorrelation and partial autocorrelation after the transformation of the integrated process using $d$-th order differentiation to express it in the form of a stationary and invertible ARMA model $(p, q)$. The time series is illustrated in the following figure (Fig. 3). The graph shows the time series to be nonstationary, but it is not clear whether it contains a seasonal component.

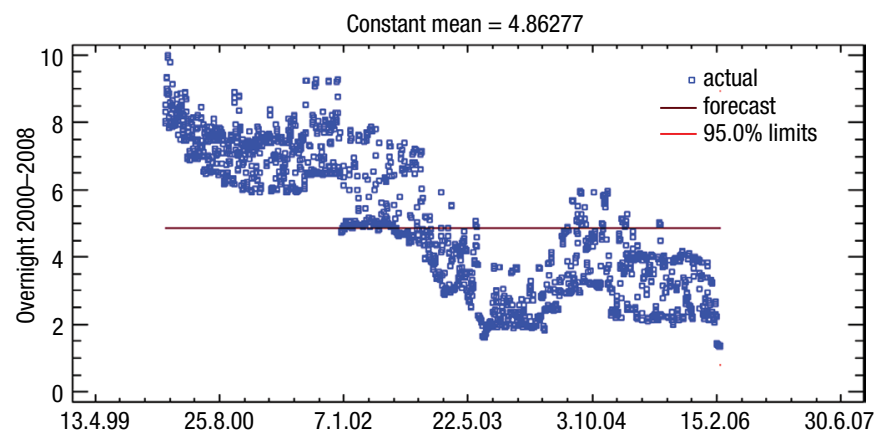

Fig. 3. Time sequence plot for time series of Overnight 2000-2008 
Time series' nonstationarity is also confirmed by the shape of the ACF and PACF. ACF values fall very slowly and the first value, as well as the PACF, is close to one. The periodogram has a significant peak in the zero (non-seasonal) frequency. Seasonality is thus not indicated by ACF, PACF, nor the periodogram.

The time series will be again stationarized by the non-seasonal difference. We will skip this step and proceed directly to the analysis of two ideal models to describe the time series. The first one is obtained after the power linearization ARIMA $(1,1,2)_{c}$, the second one after the logarithmic linearization of the original time series SARIMA $(1,1,2)$ $(1,1,1) \mathrm{c}^{20}$.

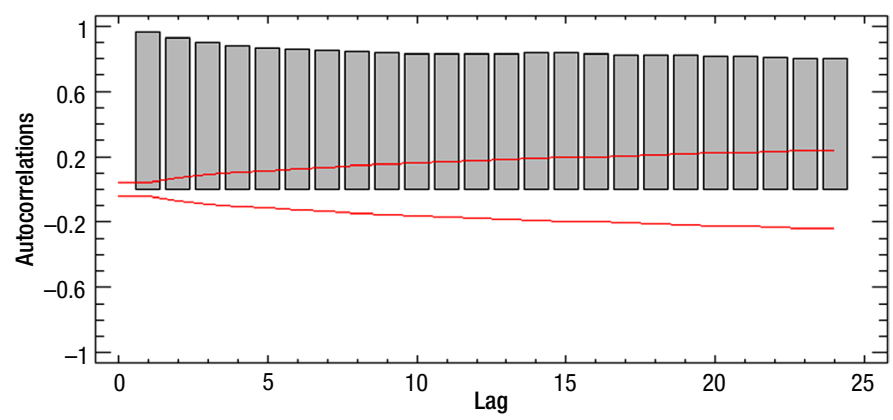

Fig. 4. Residual autocorrelations for time series of Overnight 2000-2008

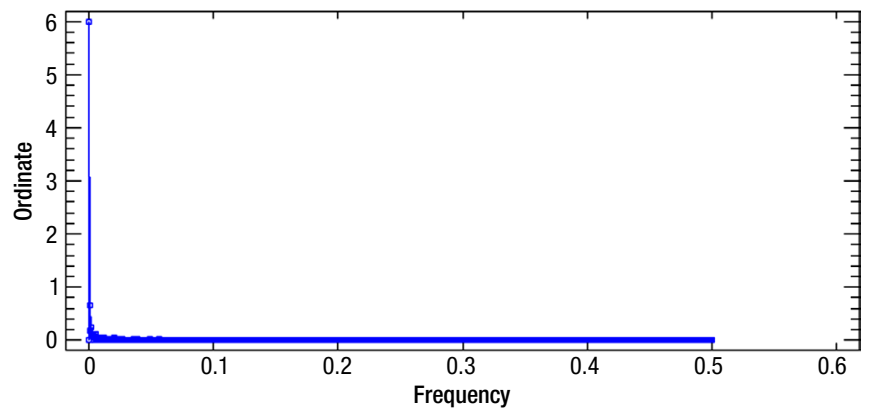

Fig. 5. Residual periodogram for time series of Overnight 2000-2008

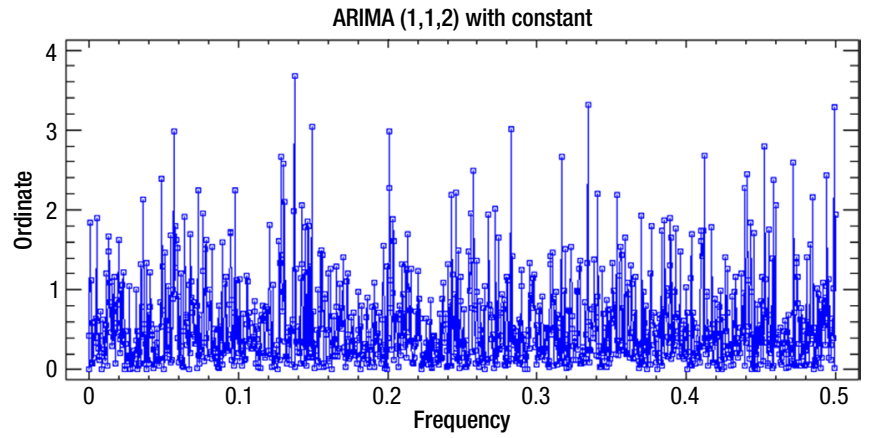

Fig. 6. Residual periodogram for adjusted time series of Overnight 2000-2008 
Residual periodogram for ARIMA model $(1,1,2)_{\mathrm{c}}$ shows residuals to be stationary. Now we focus on the extended SARIMA model $(1,1,2)(1,1,1))_{c} 20$, followed by tables of estimates and interpolation criteria as well as estimates of the model's parameters.

Interpolation criteria suggest SARIMA model $(1,1,2)(1,1,1))_{\mathrm{c}} 20$ is much more suitable than ARIMA model $(1,1,2)_{\mathrm{c}}$ for the description of the time series.

Table 2. Interpolation criteria estimates of SARIMA model $(1,1,2)(1,1,1){ }_{\mathrm{c}} 20$

\begin{tabular}{lcc}
\hline & Estimation & Validation \\
\hline Statistic & Period & Period \\
\hline RMSE & 0.536932 & 0.145012 \\
\hline MAE & 0.327448 & 0.140548 \\
\hline MAPE & 7.23435 & 10.2761 \\
\hline ME & 0.0380698 & -0.140548 \\
\hline MPE & -0.398906 & -10.2761 \\
\hline
\end{tabular}

Table 3. Parameters estimates of SARIMA model $(1,1,2)(1,1,1)_{\mathrm{c}} 20$

\begin{tabular}{lllll}
\hline Parameter & Estimate & Stand. error & $\mathrm{T}$ & P-value \\
\hline AR $(1)$ & 0.752851 & 0.021549 & 34.9367 & 0.000000 \\
\hline MA(1) & 0.850656 & 0.0291219 & 29.2102 & 0.000000 \\
\hline MA(2) & 0.112643 & 0.0264917 & 4.25202 & 0.000021 \\
\hline SAR(1) & 0.046946 & 0.021776 & 2.15587 & 0.031094 \\
\hline SMA(1) & 0.974709 & 0.001605 & 607.074 & 0.000000 \\
\hline Mean & -0.00000 & 0.0000172 & -0.420 & 0.674417 \\
\hline Constant & -0.00000 & & & \\
\hline
\end{tabular}

\section{Box-Pierce test}

Test based on first 24 autocorrelations:

Large sample test statistic $=23.3012$;

$\mathrm{P}$-value $=0.224336, \mathrm{AIC}=-1.25429$.

Figure 7 shows graphs with the time series forecast, autocorrelation and partial autocorrelation functions of the unsystematic component for estimated model and residual periodogram.

According to Akaike information criteria, the Box-Pierce test of autocorrelation of unsystematic component and interpolation criteria, SARIMA model $(1,1,2)(1,1,1)_{\mathrm{c}} 20$ appears to be better for the description of the time series compared to ARIMA model $(1,1,2)_{\mathrm{c}}$. The following figures (Figs 8,9) illustrate the residual ACF and PACF of the estimated model. P-value of the Box-Pierce test and both graphs indicate non-autocorrelation of unsystematic component, thus the estimated model appears to be correct. 




Fig. 7. Time sequence plot for time series of Overnight 2000-2008

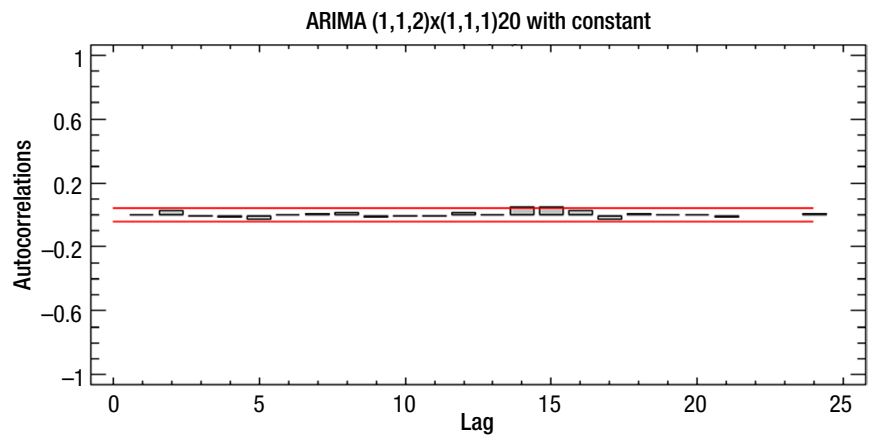

Fig. 8a. Residual autocorrelations for adjusted time series of Overnight 2000-2008

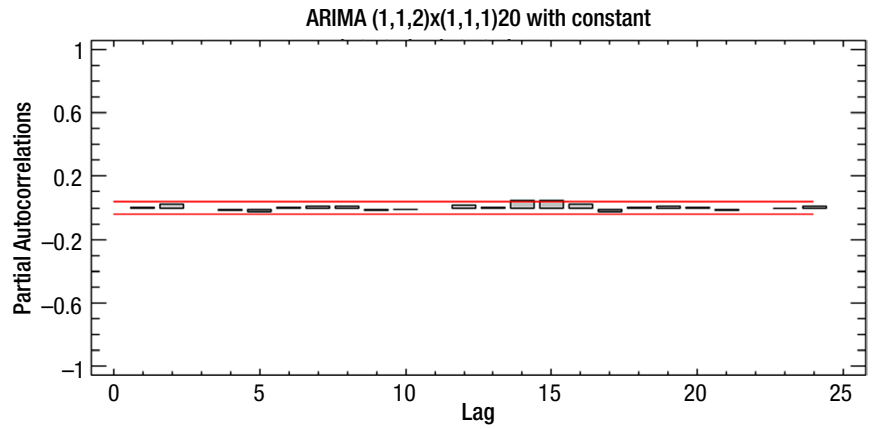

Fig. 8b. Residual partial autocorrelations for adjusted time series of Overnight 2000-2008

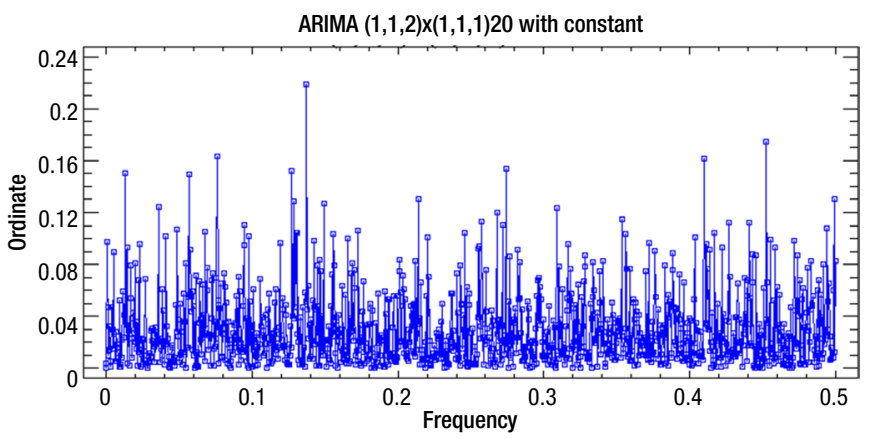

Fig. 9. Residual periodogram for adjusted time series of Overnight 2000-2008 
Now that we have information about the process from the statistical model, it can be used to construct control charts for detecting mean changes. The detection will be demonstrated on the time series of Overnight values of the Slovak crown during the period of 20 January 2000 - 17 February 2003.

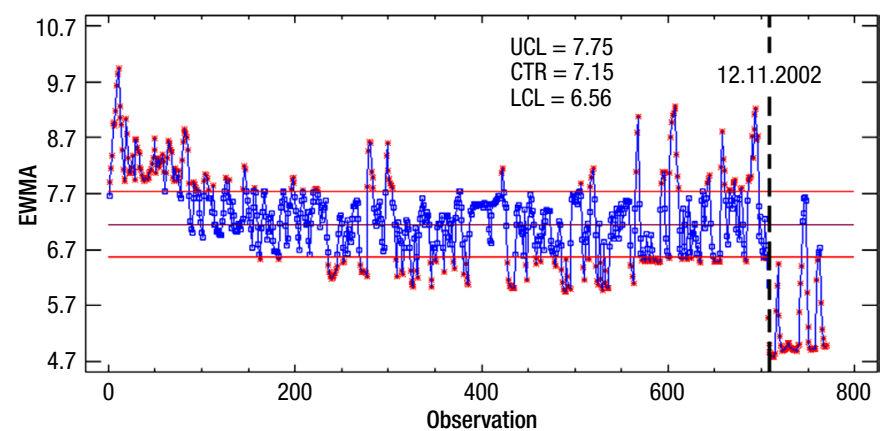

Fig. 10. EWMA chart for time series of the Overnight Slovak crown during the period of 20 January 2000 - 17 February 2003

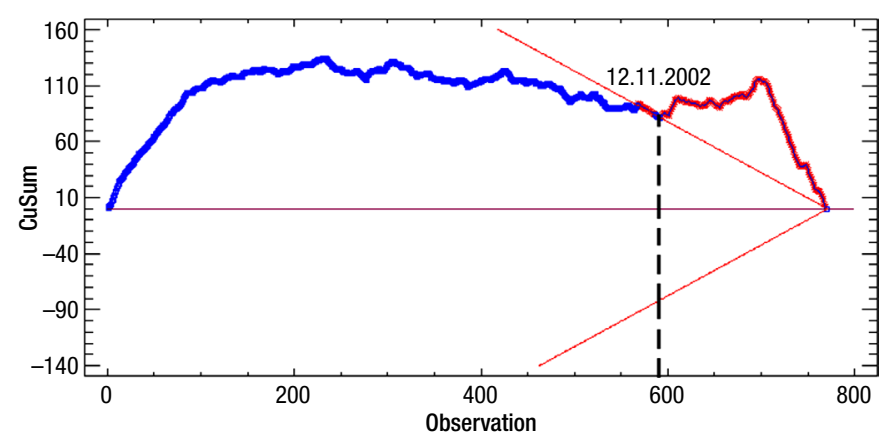

Fig. 11. CUSUM chart for time series of the Overnight Slovak crown during the period of 20 January 2000 - 17 February 2003

As evident from the previous images, the EWMA control chart and CUSUM were able to detect changes in the mean almost immediately. Both being control charts with memory, therefore information on variability in the time series of SARIMA model $(1,1,2)$ $(1,1,1)$ c 20 was used for parameter estimation to construct the diagrams very effectively. These control charts detected a shift of mean and therefore high heteroscedasticity in 12. 11. 2002, which corresponds to fluctuations of Slovak crown.

\subsection{Case study No 2: Use of EWMA and ARIMA control charts}

Using Box-Jenkins methodology, we will attempt to find a time-series model of annual Argentinian Gross Domestic Product, available as a basic index from 1951 to 1998 $(1995=100)$. Based on the model, predictions of the time series of 4 observations will be calculated and from on the ARIMA model selected, a control chart constructed.

The time series is shown in the previous figure (Fig. 12). It is obvious the time series is non-stationary, a fact also confirmed by the shape of ACF and PACF (Fig. 13) and periodogram (Fig. 14). 


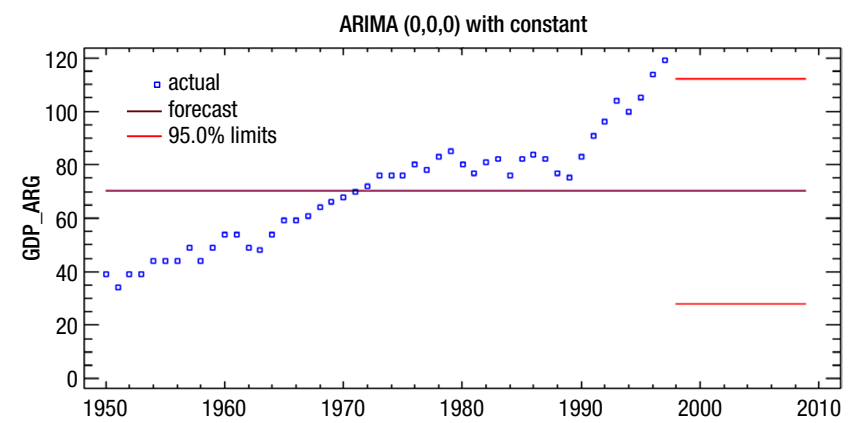

Fig. 12. Time sequence plot for time series of annual Argentinian GDP

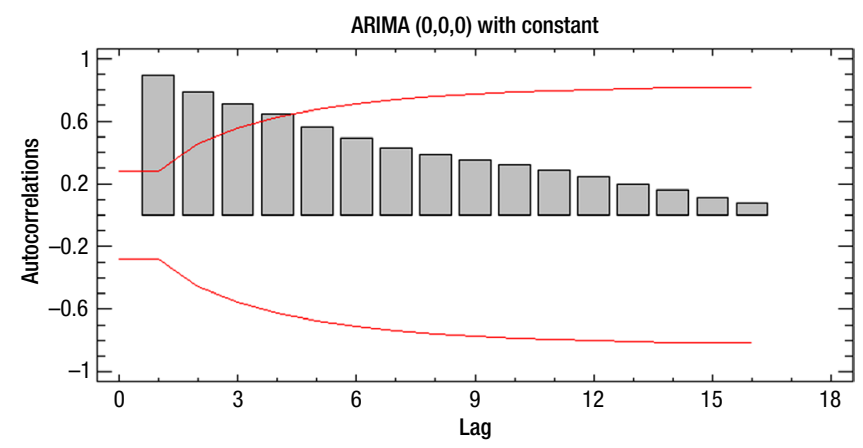

Fig. 13a. Residual autocorrelations for time series of annual Argentinian GDP

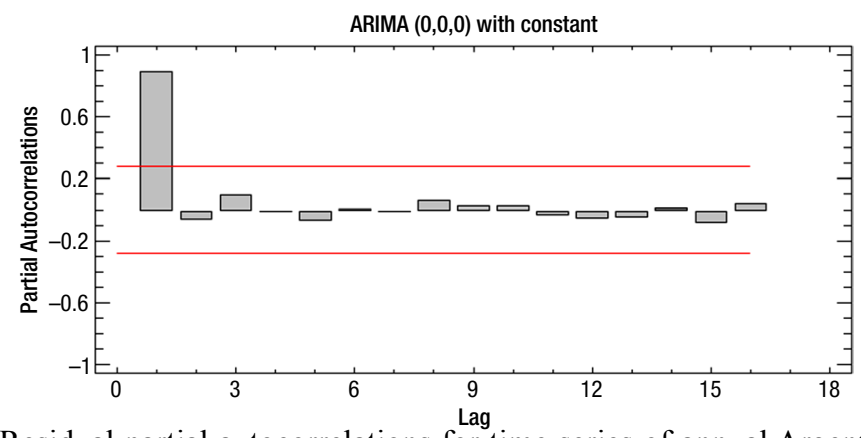

Fig. 13b. Residual partial autocorrelations for time series of annual Argentinian GDP

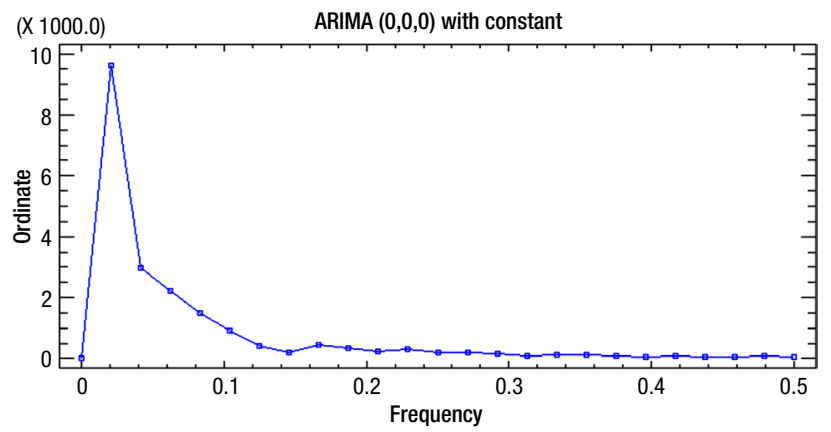

Fig. 14. Residual periodogram for time series of annual Argentinian GDP 
The values of ACF decreases slowly and the first value of the ACF and PACF is close to one. Periodogram exhibits significant peak at zero frequency, suggesting the time series is of Type I (1). We will therefore stationarize it using first differences.

Table 4. ARIMA $(0,1,0)_{\mathrm{c}}$ model input table

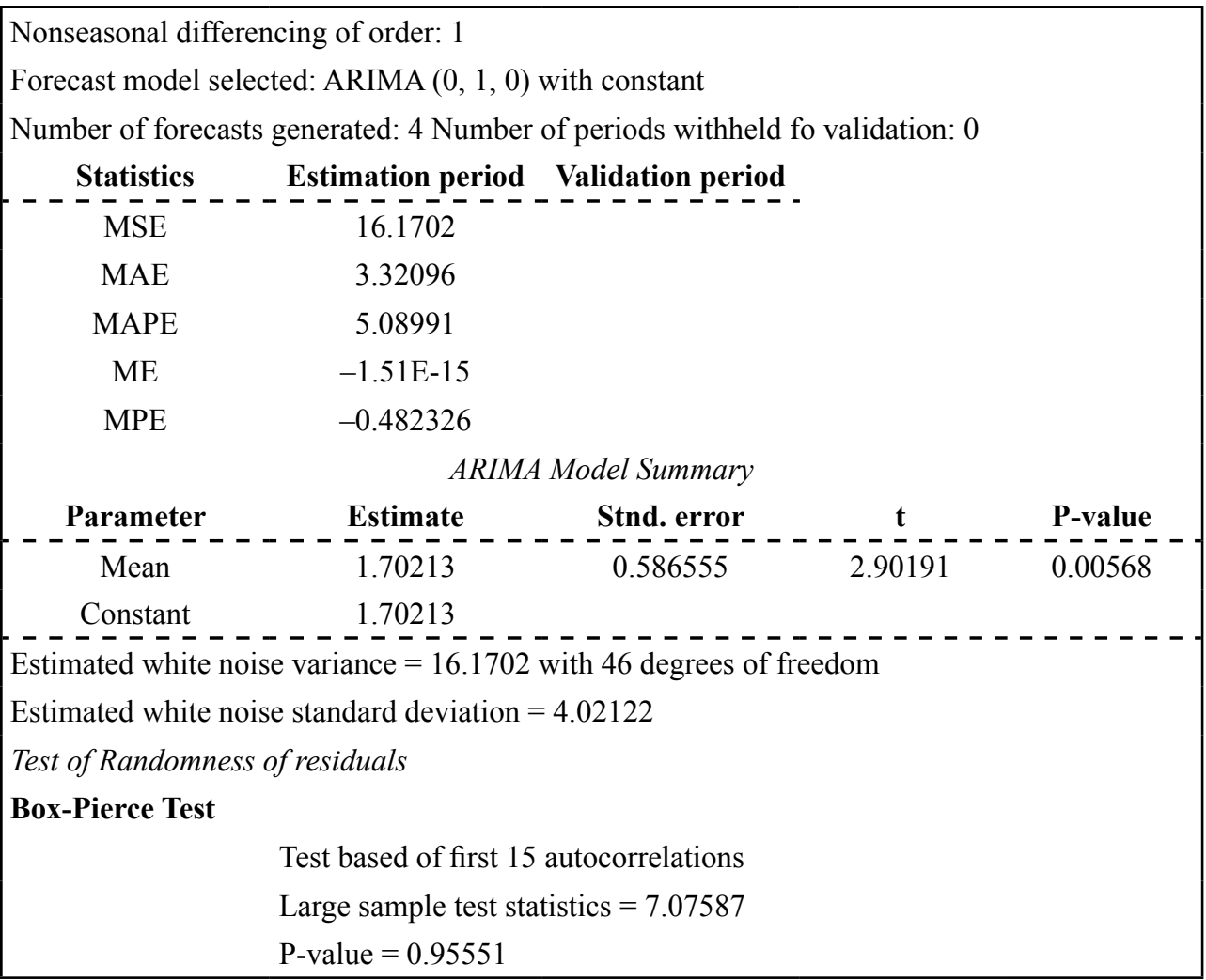

Table 4 contains characteristics of the estimated model ARIMA $(0,1,0)_{c}$ of the form:

$$
(1-\mathrm{B}) y_{t}=1.70213+a_{t}
$$

It can be expressed as:

$$
y_{t}=1.70213+y_{t-1}+a_{t}
$$

At the same time we will test whether the constant $\mathrm{c}=\mu$ is statistically significant, i.e., different from zero. The t-test result for the mean $\mu$ is also given on Figure 10 and compared to "P-value" $(0.005675)$ with significance level $\alpha(0.05)$ proves the mean and constants are different from zero. Autocorrelation test of unsystematic component is performed using the Box-Pierce test in the bottom of Figure 10. High "P-value" of this test (0.95551) indicates the unsystematic component is a type of white noise.

The conclusion is confirmed by the graph of residuals and residual periodogram (Fig. 15), as well as by residual ACF and PACF (Fig. 16) whose values lie within the tolerance limits. 


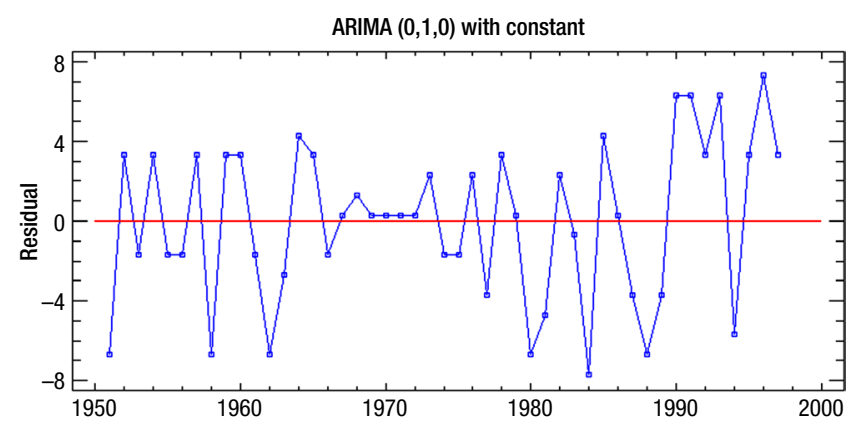

Fig. 15a. Residual plot for adjusted time series of annual Argentinian GDP

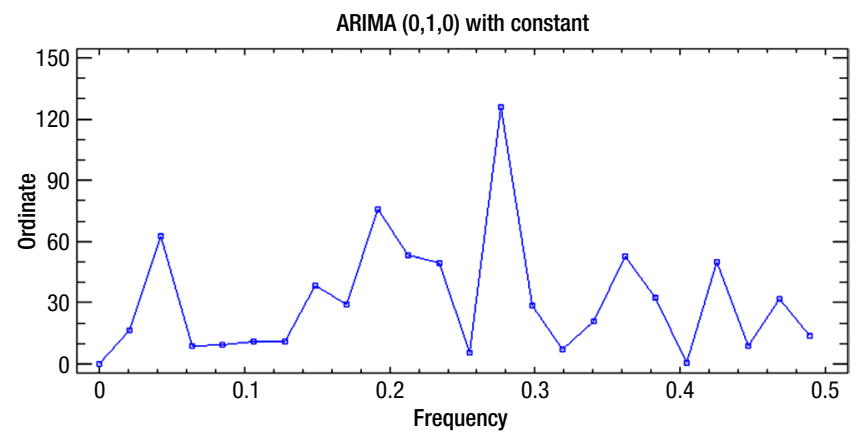

Fig. 15b. Residual periodogram for adjusted time series of annual Argentinian GDP

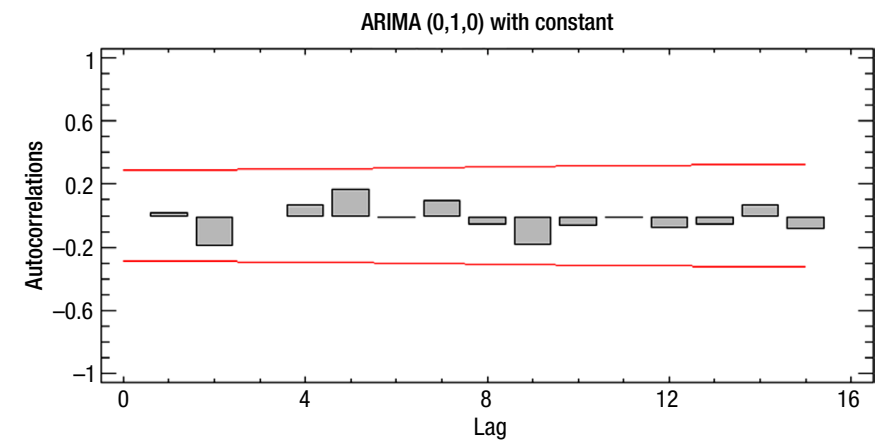

Fig. 16a. Residual autocorrelations for adjusted time series of annual Argentinian GDP

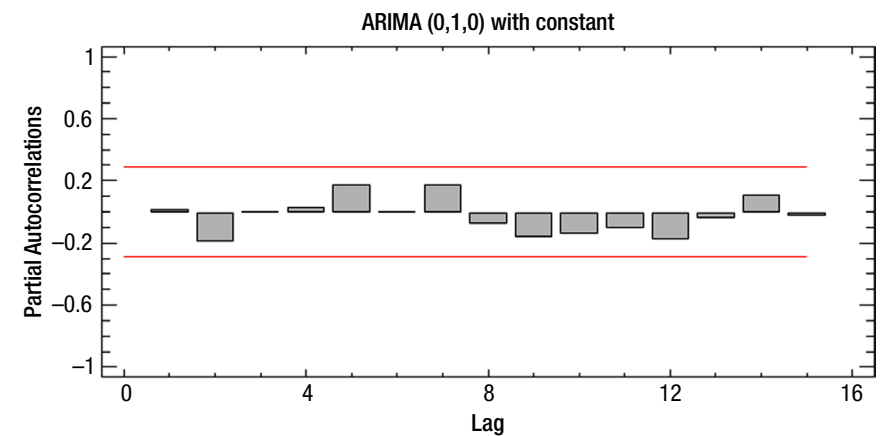

Fig. 16b. Residual partial autocorrelations for adjusted time series of annual Argentinian GDP 
We identified the time series model in the shape of random walk. If we also use it for calculation of predictions, point and interval forecasts for measurements from 49 to 53 can be obtained (Table 5).

Table 5. ARIMA $(0,1,0)_{\mathrm{c}}$ model forecasts

\begin{tabular}{|c|c|c|c|}
\hline \multicolumn{4}{|c|}{ Model: ARIMA $(0,1,0)$ with constant } \\
\hline Period & Forecast & Lower $95,0 \%$ limit & Upper $95,0 \%$ limit \\
\hline 49.0 & 120.702 & 112.608 & $128 . \overline{796}$ \\
\hline 50.0 & 122.404 & 110.957 & 133.851 \\
\hline 51.0 & 124.106 & 110.087 & 138.126 \\
\hline 52.0 & 125.809 & 109.62 & 141.997 \\
\hline 53.0 & 127.511 & 109.411 & 145.61 \\
\hline
\end{tabular}

Graph of predictions and the original time series chart with smoothed values and predictions are depicted on Figures 17 and 18.

Examples of control charts for individual values and classical EWMA chart applied to ARIMA model's residuals are in Figure 19. Based on the results of selected tests it can be assumed the residuals have normal distribution, constant variance, and are not autocorrelated. Because it can be further assumed the model's residuals have properties the controlled, variable must meet for the classic Shewhart's control chart of individual values to be applied, it is now possible to calculate residuals of regulatory limits, central line and construct an ARIMA control chart.

We can determine the process can be regarded statistically stable (no point is outside the control limits) and thus the limits can be used to verify process' statistical stability in the next period. The control charts detected a mean shift and therefore high heteroscedasticity in 1990. During the 1990s, Argentina's financial system was consolidated and strengthened. Deposits grew from less than US\$15 billion in 1991 to over US\$80 billion in 2000 while outstanding credit ( $70 \%$ of it to the private sector) tripled to nearly US\$100 billion.

\section{Discussions}

Most traditional control charting procedures are based on the assumption the process observations being monitored are independent and identically distributed (IID). With the advent of high-speed data collection schemes, the assumption of independence is usually violated, i.e., autocorrelation among measurements becomes an inherent characteristic of a stable process. Such autocorrelation causes significant deterioration in control charting performance. In order to address this, several approaches for handling autocorrelated processes have been proposed, the most popular one utilizing either Shewhart, CUSUM or EWMA chart of the residuals of the appropriately fitted ARMA model. However, procedures of this type demonstrate poor sensitivity especially when dealing with positively autocorrelated processes. As an alternative, we have explored 


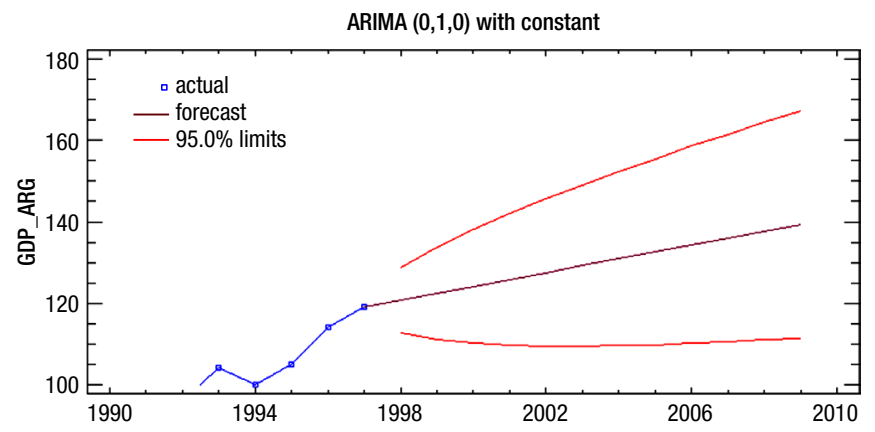

Fig. 17. Forecast plot for time series of annual Argentinian GDP

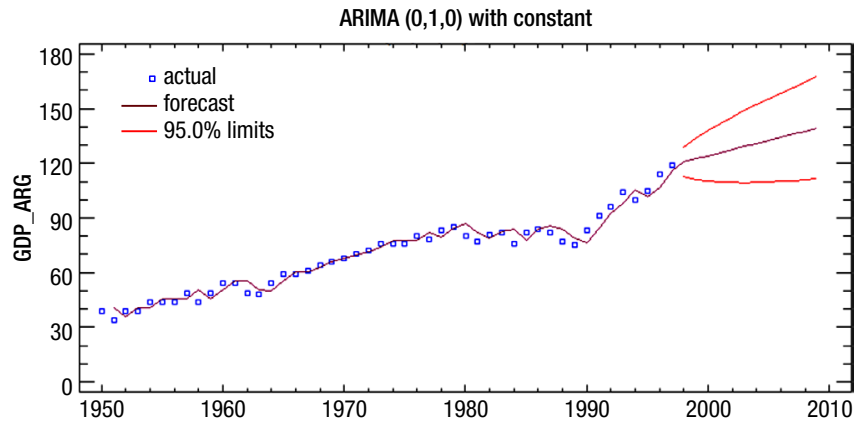

Fig. 18. Time sequence plot for time series of annual Argentinian GDP

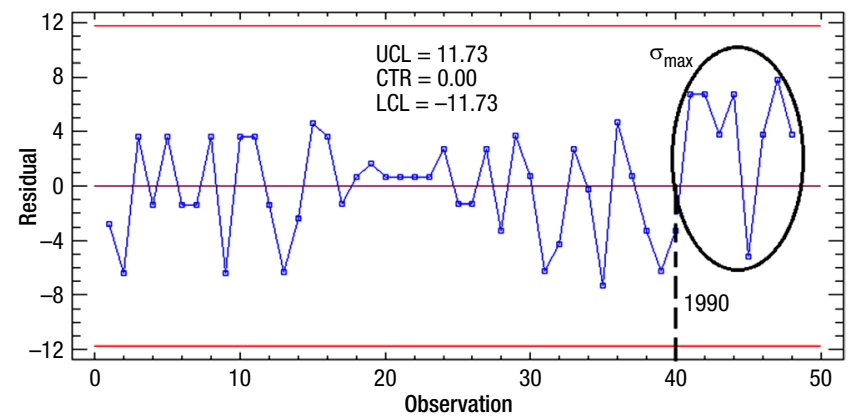

Fig. 19a. ARIMA residual chart for time series of annual Argentinian GDP

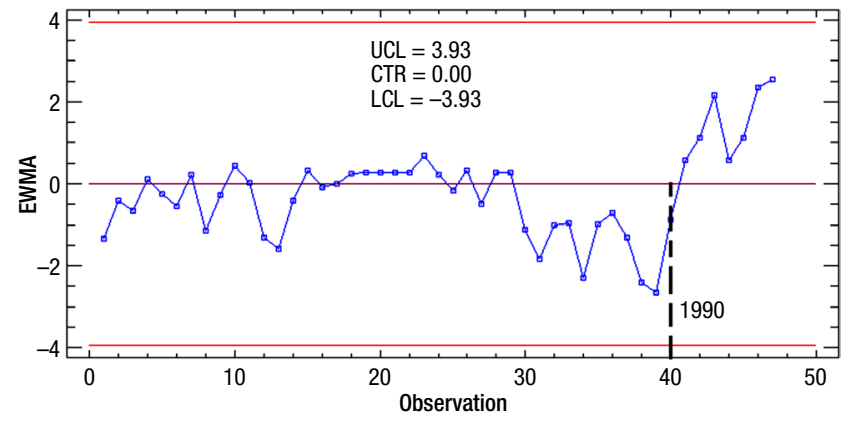

Fig. 19b. EWMA chart for Residuals for time series of annual Argentinian GDP 
the application of the statistics used in a time series procedure for detecting outliers and level shifts in process monitoring (Grubbs 1969). The study focused on the detection of level shifts of autocorrelated processes with particular emphasis on the AR (1) model. The results presented showed time series charts to be sensitive when detecting small shifts and we utilized the fact they can be used in certain situations where the data are autocorrelated.

\section{Conclusions}

The paper dealt with the regulation charts applications in financial data. This kind of data is very sensitive to mean shifting and strong autocorrelation appears very often. Therefore we put a focus on dynamic regulation charts CUSUM, EWMA and ARIMA models. We highlighted the versatility of control charts not only in manufacturing, but also in managing the financial stability of financial flows in this paper. A refined identification of the type of intervention affecting the process will allow users to effectively track the source of an out-of-control situation which is an important step in eliminating the special causes of variation. It is also important to note that the proposed procedure can also be applied when dealing with a more general autoregressive integrated moving average model.

Autocorrelated process observations mainly arise under automated data collection schemes, typically controlled by software which can be upgraded to handle SPC functions. Under such integrated scheme, the usefulness of the proposed procedure will be optimized. Based on information from chapter 3, we would recommend a properly designed time series control charts as control charts for individual measurements in a wide range of applications. These are almost perfectly nonparametric (distribution-free) procedures.

\section{References}

Atienza, O. O.; Tang, L. C.; Ang, B. W. 1997. ARL properties of a sample autocorrelation chart, Computers and Industrial Engineering 33(3-4): 733-736.

http://dx.doi.org/10.1016/S0360-8352(97)00234-9

Alwan, L. C. 1992. Effects of autocorrelation on control chart performance, Communications in Statistics - Theory and Methods 21(4): 1025-1049. http://dx.doi.org/10.1080/03610929208830829

Apley, D. W.; Tsung, F. 2002. The autoregressive $\mathrm{T}^{2}$ chart for monitoring univariate autocorrelated processes, Journal of Quality Technology 34(1): 80-96.

Chambers, D. S.; Wheeler, D. J. 1992. Understanding statistical process control. 2nd ed. Tennessee: SPC Press, Inc.

Chandra, M. J. 2001. Statistical quality control. Florida: CRC Press, LLC.

Dyer, J. N.; Conerly, D. M.; Adams, B. M. 2003. A simulation study and evaluation of multivariate forecast based control charts applied to ARMA processes, Journal of Statistical Computation and Simulation 73(10): 709-724. http://dx.doi.org/10.1080/0094965031000062168

Gervini, D. 2003. A robust and efficient adaptive reweighted estimator of multivariate location and scatter, Journal of Multivariate Analysis 84(1): 116-144.

http://dx.doi.org/10.1016/S0047-259X(02)00018-0

Grubbs, F. E. 1969. Procedures for detecting outlying observations in samples, Technometrics 11(1): 1-21. http://dx.doi.org/10.1080/00401706.1969.10490657 
Harris, T. J.; Ross, W. H. 1991. Statistical process control procedures for correlated observations, Canadian Journal of Chemical Engineering 69(1): 48-57.

http://dx.doi.org/10.1002/cjce.5450690106

Hušek, R. 2007. Ekonometrická analýza [Econometric analysis]. Praha: Oeconomica.

Kovarik, M.; Kral, M. 2011. Carry trade. 1st ed. Zilina: Georg, Inc.

Kovarik, M. 2012. Usage of control charts and stochastic differential equations for volatility change point detection in time series. 1st ed. Zilina: Georg, Inc.

Kovarik, M. 2013a. Research in the field of monitoring autocorrelated processes using of control charts. 1st ed. Zilina: Georg, Inc.

Kovarik, M. 2013b. Volatility change point detection using stochastic differential equations and time series control charts, International Journal of Mathematical Models and Methods in Applied Sciences 2(7): 121-132.

Kovarik, M.; Klimek, P. 2013. Survey and analysis of the use statistical process control methods in selected Czech manufacturing companies, International Journal of Mathematical Models and Methods in Applied Sciences 4(7): 358-369.

Kovarik, M.; Sarga, L. 2014. Implementing control charts to corporate financial management, WSEAS Transactions on Mathematics 13: 246-255.

Krieger, C. A.; Champ, C. W.; Alwan, L. C. 1992. Monitoring an autocorrelated process. Presented at the Pittsburgh Conference on Modeling and Simulation, Pittsburgh, PA.

Lu, C. W.; Reynolds, M. R. 1999a. EWMA control charts for monitoring the mean of autocorrelated processes, Journal of Quality Technology 31(2): 166-188.

Lu, C. W.; Reynolds, M. R. 1999b. Control charts for monitoring the mean and variance of autocorrelated processes, Journal of Quality Technology 31(3): 259-274.

Meloun, M.; Militký, J. 2006. Kompendium statistického zpracování dat [Statistical data processing compendium]. Praha: Academia.

Montgomery, D. C, Friedman, D. J. 1989. Statistical process control in computer integrated manufacturing environment, in J. B. Keats, N. F. Hubele (Eds.). Statistical process control in automated manufacturing. New York: Marcel Dekker.

Montgomery, D. C.; Mastrangelo, C. M. 1991. Some statistical process control methods for autocorrelated data, Journal of Quality Technology 23(3): 179-204.

Noskievičová, D. 2008. Vybrané metody statistické regulace procesu pro autokorelovaná data [Selected methods for statistical process regulation with autocorrelated data], AUTOMA 9(10): 40-43.

Runger, G. C.; Willemain, T. R. 1996. Batch-means control charts for autocorrelated data, IIE Transactions 28(6): 483-487.

Sun, J.; Xu, L. 2004. Batch average control chart, ASQ Annual Quality Congress Proceedings, 24-26 May 2004, Toronto, Ontario 58: 85-96.

Yourstone, S. A.; Montgomery, D. C. 1991. Detection of process upsets - sample autocorrelation control chart and group autocorrelation control chart applications, Quality and Reliability Engineering International 7(3): 133-140. http://dx.doi.org/10.1002/qre.4680070304 
Martin KOVÁŘíK, PhD, graduated at the Faculty of Management and Economics, Tomas Bata University in Zlin, where he is lecturing at the Department of Statistics and Quantitative Methods since 2009. He also graduated at Faculty of Applied Informatics, Tomas Bata University in Zlin in the field of Information Technology. Author and co-author of 7 books and 5 lecture notes, his research is focused on mathematical and statistical methods in quality management and computationally-intensive statistical data analyses with results published in numerous peer-reviewed journals and presented at conferences in the Czech Republic as well as internationally. Martin Kováŕík is also a consultant of statistical data analysis, application of statistical methods in quality management and questionnairebased surveys data processing.

Libor SARGA, Ing., is a PhD candidate at the Department of Statistics and Quantitative Methods, Faculty of Management and Economics, Tomas Bata University in Zlin, Czech Republic. His professional interests include information technology, data security policies, and quantitative data processing. His dissertation will focus on setting a suitable data security framework in organizations.

Petr KLÍMEK, Associate Professor, is a university teacher and a scientific researcher currently with the Department of Statistics and Quantitative Methods, Faculty of Management and Economics, Tomas Bata University in Zlín. He is the author or co-author of many publications in the field of statistical data analysis in books, teaching scripts, and scientific articles. 